\title{
A fourth component in the young multiple system V 773 Tauri ${ }^{\star}$
}

\author{
J. Woitas ${ }^{\star}$ \\ Thüringer Landessternwarte Tautenburg, Sternwarte 5, 07778 Tautenburg, Germany \\ Received 8 November 2002 / Accepted 19 May 2003

\begin{abstract}
I report on a new component in the pre-main sequence multiple system V 773 Tauri. This second visual companion, V 773 Tau C, with a projected separation of $\approx 0$ ' 2 has been detected using speckle interferometry in the near-infrared. Repeated observations from 1996 to 2002 show significant orbital motion and thus confirm the character of the new companion as a gravitationally bound star. Together with the two components of the spectroscopic binary V 773 Tau A and the previously known visual companion V 773 Tau B, the V 773 Tau system appears as a young "mini-cluster" of four T Tauri stars within a sphere of a radius less than $100 \mathrm{AU}$. V 773 Tau A, B and C form a triple system that is not hierarchic, but is apparently stable despite of this. The brightness of V 773 Tau $\mathrm{C}$ has probably increased over the last years, which may explain its non-detection in previous binary surveys.
\end{abstract}

Key words. stars: binaries: general - stars: individual: V 773 Tau - stars: pre-main sequence - techniques: interferometric

\section{Introduction}

High angular resolution surveys for companions to $\mathrm{T}$ Tauri stars in nearby star forming regions (SFRs) and young clusters (cf. Mathieu et al. 2000 for a review) have revealed that they show a companion frequency at least as high as among $\mathrm{G}$ and $\mathrm{K}$ dwarfs in the solar neighbourhood (50-60\%, e.g. Duquennoy \& Mayor 1991). This means that multiplicity must be established already at a very early phase of stellar evolution, and that binary formation is not an exception but perhaps the dominant mode of star formation. The probably most surprising outcome of these binary surveys is that one of the nearest SFRs, the Taurus-Auriga association, shows a significant binary overabundance compared with main-sequence dwarfs in the solar neighbourhood and also most other samples of young stars (Köhler \& Leinert 1998, and references therein). If the distribution of orbital periods in Taurus-Auriga is the same as for main sequence binaries, almost all young stars in this SFR will be members of binary or multiple systems.

Although all known pre-main sequence stars in TaurusAuriga have been targets of systematic binary surveys, some companions were apparently overlooked. Almost all of them are components of triple or higher order multiple systems (Duchêne 1999; Richichi et al. 1999). In this paper I report on a previously unknown third companion to V 773 Tau.

V 773 Tau is one of the optically brightest members of the Taurus-Auriga association, and is also a strong radio source. The latter property allowed a precise determination of its distance, which is $148 \pm 5 \mathrm{pc}$, using VLBI astrometry

* Based on observations collected at the German-Spanish Astronomical Center on Calar Alto, Spain.

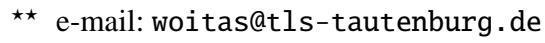

(Lestrade et al. 1999). V 773 Tau is classified as a weak-lined $\mathrm{T}$ Tauri star regarding its $\mathrm{H} \alpha$ equivalent width of $4 \AA$ (Herbig \& Bell 1988).

Leinert et al. (1993) and Ghez et al. (1993) have detected a visual companion to $\mathrm{V} 773$ Tau with a projected separation of $\approx 0$.' 15 using speckle interferometry in the $K$-band. Since this companion, hereafter named V 773 Tau B, was close to its periastron at the time of detection, Tamazian et al. (2002) could derive a first orbital solution based on 9 data points that cover $\approx 160^{\circ}$ in position angle, indicating a dynamical system mass of $3.20 \pm 0.71 M_{\odot}$. Welty (1995) has shown that the main component, V 773 Tau A, is itself a spectroscopic binary with an orbital period of 51 days. Duchêne et al. (2001) have briefly announced the possible presence of a fourth close component in the V 773 Tau system. In this paper astrometric and photometric properties of this object are given, and its physical membership to V 773 Tau is confirmed from its orbital motion.

\section{Observations and data analysis}

V 773 Tau has been observed with the near-infrared camera Omega Cass at the $3.5 \mathrm{~m}$-telescope on Calar Alto in February 2001, November 2001 and October 2002. All observations were carried out in the $K$-band at $\lambda=2.2 \mu \mathrm{m}$. Omega Cass is in its subarray mode capable of taking fast sequences of short exposures with integration times $\tau \approx 0.1 \mathrm{~s}$. This allows the application of speckle interferometry as a high angular resolution technique that yields information about object structures down to the diffraction limit that is $\lambda / D \approx 0$.' 13 for the given instrumentation. If it is known from previous observations that the object is a binary, then its parameters can be reliably determined even down to a projected separation of $\lambda /(2 D)$. 
In each observing run at least 1500 short exposures were taken for V 773 Tau as well as for the nearby single star SAO 76511 that is used as PSF calibrator. These exposures are stored as "data cubes" of 250 frames. After each data cube the telescope position is switched between the object and the PSF calibrator. This ensures that both are observed at nearly the same airmass and seeing conditions. The mean power spectrum of one data cube for the object is deconvolved with that for the reference star. The phase is reconstructed using the algorithm by Knox \& Thompson (1974) and the bispectrum method (Lohmann et al. 1983). Finally, modulus and phase of the complex visibility are averaged over all data cubes. These mean complex visibilities, derived from all observations of V 773 Tau within one night, are used to determine the parameters of the visual companions, i.e. their position angles, projected separations and flux ratios with respect to the primary. For this purpose artificial complex visibilities are constructed from sets of triple star models and fit to the data ${ }^{1}$. As an example data, model and the residuals of the fit are shown for the observation at 19 Oct. 2002 in Fig. 1. The stripe patterns (from upper right to lower left in the images) are the typical sign of a stellar companion in Fourier space. Note also the shading at the right and left hand sides of the images. This is caused by the presence of V 773 Tau B that is resolved although its projected separation with respect to V 773 Tau A is only $0 . ' 115$.

The pixel scale is $\approx 00^{\prime} 095 /$ pixel for Omega Cass and was, as well as the detector orientation, empirically determined for the individual observations using astrometric fits to images of the Orion Trapezium cluster core where precise astrometry has been given by McCaughrean \& Stauffer (1994).

In analyzing the data one has to consider that the Nyquist frequency, defined as half the sampling frequency

$f_{\mathrm{N}}=f_{\mathrm{s}} / 2=\frac{1}{2 \times 0.095}=5.3 \operatorname{arcsec}^{-1}$

is less than the cutoff frequency

$f_{\mathrm{c}}=\frac{D}{\lambda}=\frac{1}{0 !^{\prime} 13}=7.7 \operatorname{arcsec}^{-1}$

for the instrument and telescope configuration used. This may cause aliasing effects for spatial frequencies larger than

$f_{\mathrm{s}}-f_{\mathrm{c}}=2.9 \operatorname{arcsec}^{-1}$.

In Fig. 1 this corresponds to a radius of $R=35$ pixel around the images centres, while the whole data range used for the fit is up to $R=64$ pixel, i.e. the Nyquist spatial frequency. This has probably not a large influence on the general picture. $\mathrm{V} 773$ Tau $\mathrm{C}$ will be resolved already at $f \leq 35$ pixel, and the relative position of $\mathrm{V} 773$ Tau $\mathrm{B}$ matches well the orbital fit by Tamazian et al. (2002) and thus seems reliable. For spatial frequencies $f>35$ pixel the signal is however affected by an unknown bias. Therefore any results about flux ratios and photometry for very close companions like V 773 Tau B and C are highly uncertain for Omega Cass data.

\footnotetext{
${ }^{1}$ A more detailed description of the data reduction and analysis process can be found in Köhler et al. (2000).

${ }^{2}$ V 773 Tau A and B were not resolved in this observation. The measured flux ratio is $F_{\mathrm{C}} / F_{\mathrm{A}+\mathrm{B}}=0.06 \pm 0.01$.
}

After detecting the new companion in the Omega Cass data, I have re-analysed near infrared speckle interferometric data of V 773 Tau obtained with the MAGIC camera also at the $3.5 \mathrm{~m}$-telescope on Calar Alto in September 1996. The pixel scale of this camera is $0 .{ }^{\prime} 07 /$ pixel, corresponding to a Nyquist frequency of $7.1 \mathrm{arcsec}^{-1}$. This means that the mentioned aliasing problem does barely exist here, and the derived binary parameters are thus almost unbiased by this effect. Evidence for the presence of V 773 Tau C could indeed be found. At the time of this observation the brighter companion V 773 Tau B had a projected separation of less than 0 .' 05 from the primary (Tamazian et al. 2002). The pair V 773 Tau AB thus appears as a point source even in a diffraction limited image, and the contribution of V 773 Tau B to the complex visibility is approximately constant for all power spectrum frequencies. The flux ratio has therefore to be read as $F_{\mathrm{C}} / F_{\mathrm{A}+\mathrm{B}}=0.06 \pm 0.01$ for this observation. This is at the edge of the dynamical range accessible with speckle methods, but as only a double star model has to be used here, the parameters of V 773 Tau $C$ can be determined from these data with sufficient accuracy.

To test the reliability of the fits I have calculated the $\chi^{2}$, defined here as

$\chi^{2}=\frac{1}{n-1} \sum_{k=1}^{n} \frac{\left(x_{\text {data }}(k)-x_{\text {model }}(k)\right)^{2}}{\sigma^{2}(k)}$,

where the sum is over all pixels used for the fit. $x$ and $\sigma$ are the values of modulus or phase and, respectively, the standard deviations for individual pixels resulting from averaging over all data cubes. While this $\chi^{2}$ is a reasonable indicator for the quality of the fit, its mathematical interpretation should be taken with care. The number of degrees of freedom is probably not $n-1$ because the pixels are correlated over a distance (in frequency space) that is defined by the reciprocal seeing width. This means that the $\chi^{2}$ values have to be multiplied with a factor that can be estimated as

$C=\frac{F O W}{\left(F W H M_{\text {seeing }}\right)^{2}}$.

The field of view $(F O V)$ is 8 ". $^{\prime} 96^{2}$ for MAGIC and $122^{\prime \prime} 16^{2}$ for the subarray of Omega Cass. In Table 1 an overview of all observations of V 773 Tau C is presented, and the corrected and uncorrected $\chi^{2}$ values are given. The latter ones indicate that for all observations the error of the fit is on average smaller than the $1 \sigma$ uncertainty of the data.

Relative astrometry and photometry is also given for V 773 Tau B for the last three observations. A complete list of all binary parameter measurements of this latter companion since 1990 can be found in Tamazian et al. (2002, Table 1 therein).

\section{Discussion}

\subsection{Reliability of the detection}

Since the projected separations of V 773 Tau B measured in and after February 2001 are in the order of both the diffraction limit and the pixel scale, one may doubt that V 773 Tau really is a visual triple star. If this were the case, the "new" companion 

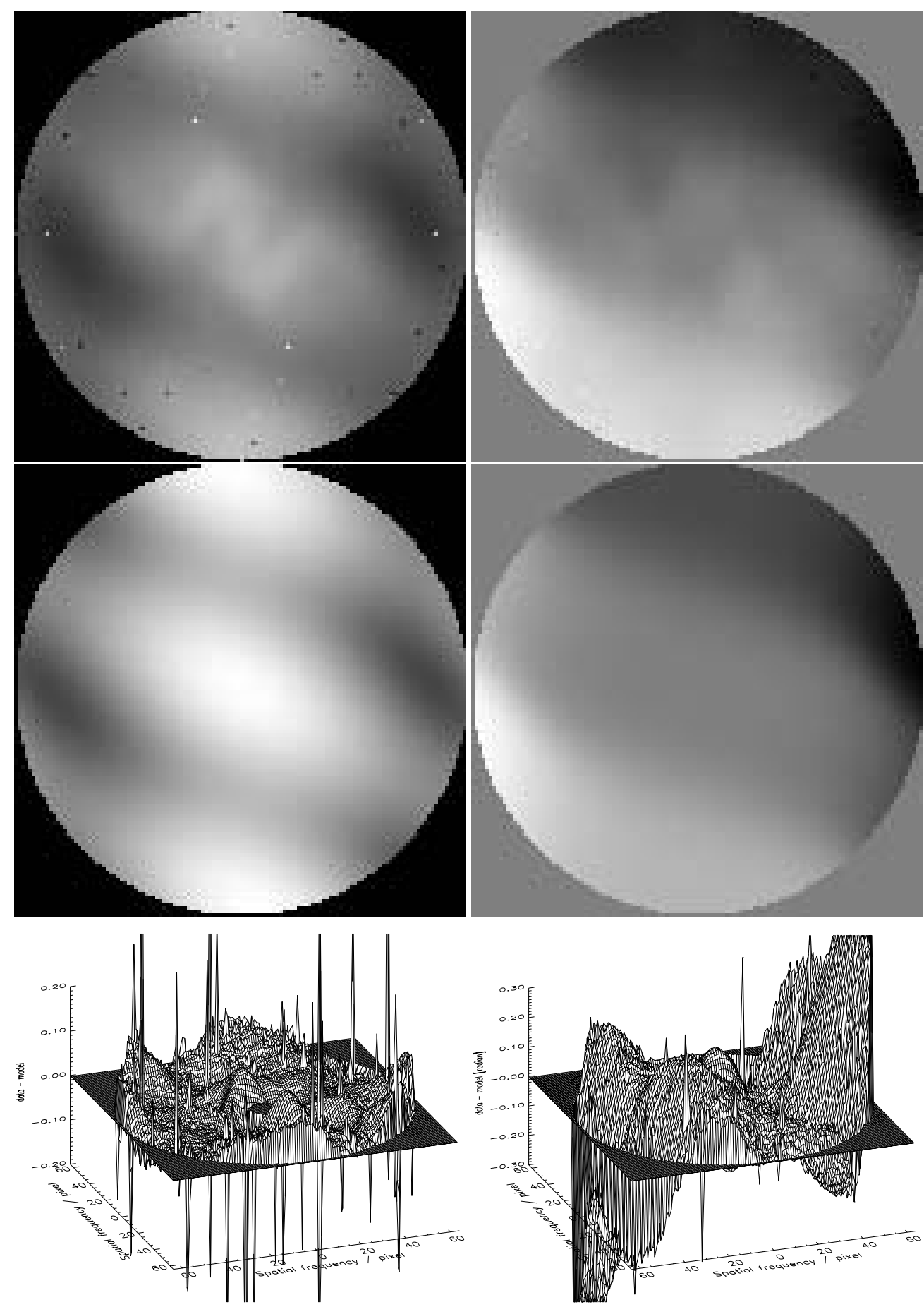

Fig. 1. First line: modulus (left) and bispectrum phase (right) of the complex visibility for V 773 Tau, derived from 3000 short $(\tau=0.13 \mathrm{~s}$ ) exposures obtained in the $K$-band with the Omega Cass camera at the $3.5 \mathrm{~m}$-telescope at Calar Alto on 19 October 2002. The images have been cut off at the Nyquist spatial frequency that is $5.3 \mathrm{arcsec}^{-1}$ for the adopted pixel scale of $0.095 /$ pixel. North is up, and east is to the left. Second line: Artificial modulus (left) and phase (right) that are constructed from a triple star model and fit to the data. Last line: residuals of the fit. The modulus is normalized to one, the phase is given in radian.

would just be V 773 Tau B that has appeared on the opposite side of the primary after passing its periastron. There are several arguments against this idea: The signature of V 773 Tau B is seen in Fig. 1 at a position different from V 773 Tau C, and assuming that V 773 Tau is a visual triple star significantly improves the model fit mentioned in Sect. 2. For the data from October 2002 presented in Fig. 1, a fit only with a double star model (i.e. without V 773 B) yields a (corrected) $\chi^{2}=4.85$ for the modulus and $\chi^{2}=112.0$ for the phase. In particular for the modulus this is about ten times larger than the value given in Table 1. The triple star solution has been reproduced in three data sets obtained at different epochs. Furthermore, the data points obtained for V 773 Tau B until 1994 and for the new companion after 1996 cannot be explained by orbital motion of 
Table 1. Relative astrometry and flux ratio of the new companion V 773 Tau C with respect to V 773 Tau Aa obtained from speckle interferometric observations at the $3.5 \mathrm{~m}$-telescope on Calar Alto. For the last observations from Feb. 2001 to Oct. 2002 also the results for component V 773 Tau B are given.

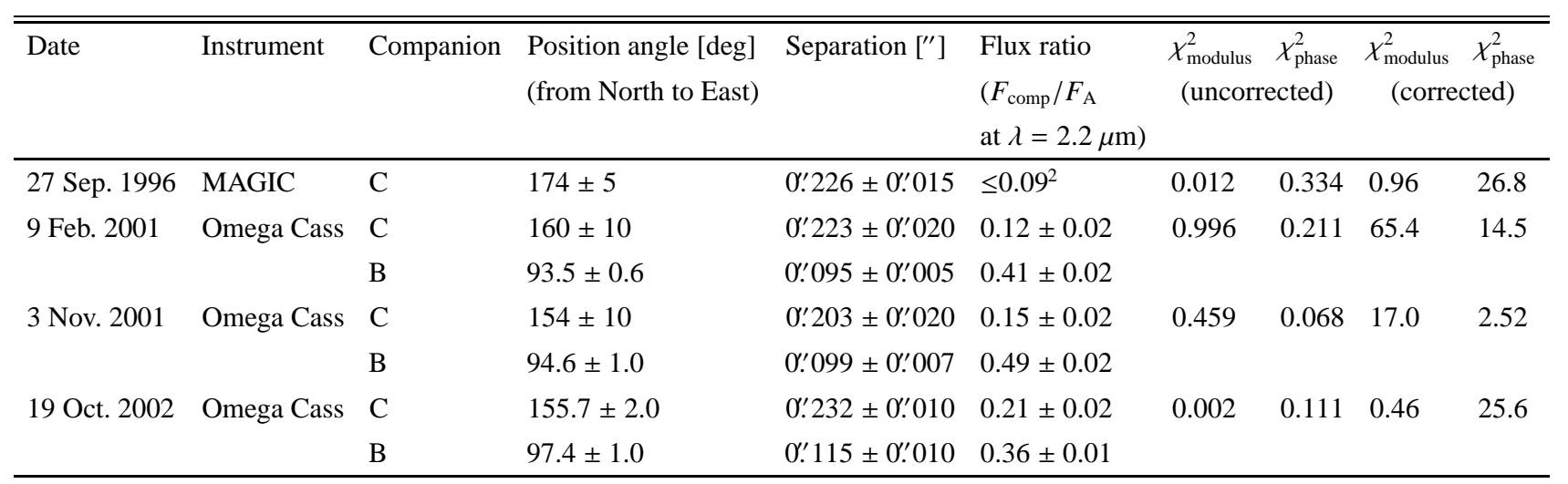

one object since such a companion would have passed through its periastron much too fast.

Another question is if $\mathrm{V} 773$ Tau C really is a gravitationally bound stellar companion. To check this, one has to prove if its relative motion with respect to the primary is orbital motion. A method for this has been discussed in detail in a previous paper (Woitas et al. 2001). Briefly, from a linear fit to the four position measurements of V 773 Tau C from 1996 to 2002, I derive a tangential velocity of $v_{\mathrm{t}}=8.7 \pm 2.6 \mathrm{~km} \mathrm{~s}^{-1}$. This means that the relative motion is significant on the $3 \sigma$ level and that modulus and direction of this motion are very well explainable with orbital motion. A background star projected by chance close to V 773 Tau A would move in a direction antiparallel to the proper motion of $\mathrm{V} 773 \mathrm{Tau}\left(\mu_{\alpha}=\right.$ $0.65 \mathrm{mas} / \mathrm{yr}, \mu_{\delta}=-24.89 \mathrm{mas} / \mathrm{yr}$ from the Hipparcos catalogue). This translates into a relative motion of a projected companion of $17.5 \mathrm{~km} \mathrm{~s}^{-1}$ in northern direction which is inconsistent with the data. Furthermore, one can estimate from the low stellar density in Taurus-Auriga that the probability to find a chance-projected object brighter than $K=12$ mag within a radius of $0 . \prime 3$ around an association member is only $1.1 \times 10^{-5}$ (Leinert et al. 1993). Although I cannot give absolute $K$-band photometry for V 773 Tau C, it is certainly brighter than $K=12 \mathrm{mag}$, given the mean system magnitude $K=6.45 \pm 0.09$ mag (Kenyon \& Hartmann 1995) and the flux ratios from Table 1 . Any random projections are thus very unlikely. As result of this discussion I conclude that there are really two visual companions in the V 773 Tau system, which makes this system a quadruple, in combination with the close spectroscopic companion to V 773 Tau A detected by Welty (1995). The fact that V 773 Tau C has not been detected in previous observations might be the outcome of a long term variability of its brightness. As discussed in Sect. 2 the flux ratios $F_{\mathrm{B}} / F_{\mathrm{A}}$ and $F_{\mathrm{C}} / F_{\mathrm{A}}$ derived from Omega Cass data are affected with a bias resulting from aliasing at higher spatial frequencies and have therefore to be taken with caution. The value $F_{\mathrm{C}} / F_{\mathrm{A}+\mathrm{B}}=0.06$ obtained with MAGIC in 1996 is however not affected with this bias. If one assumes $F_{\mathrm{B}} / F_{\mathrm{A}} \leq 0.5$, which seems justified looking at Table 1 and also at the (unbiased) value of $F_{\mathrm{B}} / F_{\mathrm{A}}=0.13 \pm 0.04$ given by Leinert et al. (1993), one ends up with $F_{\mathrm{C}} / F_{\mathrm{A}} \leq 0.09$ for this observation. On the other hand, the value for the observation in Oct. 2002 is indeed around $F_{\mathrm{C}} / F_{\mathrm{A}} \approx 0.2$. This can be proven by lowering the fit radius to $f=35$ pixel where the bias disappears. Since V 773 Tau is a weak-lined T Tauri star with no signs of active circumstellar accretion, variable extinction might explain this unexpected strong variability.

\subsection{The V 773 Tauri "mini-cluster"}

The real (i.e. de-projected) separation between V 773 Tau C and V 773 Tau A cannot be determined from my data. The presence of measurable orbital motion however indicates that this separation is much less than $100 \mathrm{AU}$. For this semimajor axis the annual orbital motion would be $0.6^{\circ} / \mathrm{yr}$ in position angle for the idealized situation of a circular orbit viewed pole-on and a system mass of $3 M_{\odot}$. The latter is similar to the dynamical system mass derived for V 773 Tau AB by Tamazian et al. (2002). The observed orbital motion is much faster, so $100 \mathrm{AU}$ is a conservative upper limit for the separation between V 773 Tau A and $\mathrm{C}$. The semimajor axis of component $\mathrm{V} 773$ Tau B is $a_{\mathrm{AB}} \approx 37 \mathrm{AU}$ (Tamazian et al. 2002). This means that together with the spectroscopic binary V 773 Tau A, the whole system consists of four stars within a sphere with a radius that is less than $100 \mathrm{AU}$. To my knowledge, no other similar "minicluster" of T Tauri stars is known at this time. The other young quadruple systems in Taurus-Auriga (GG Tau, UZ Tau, UX Tau and $\mathrm{BD}+26718 \mathrm{~B}$ ) consist of components separated by several arcseconds. The very small size of the V 773 Tau system strongly indicates that all components have formed from the same molecular cloud core and thus must be coeval to a high degree. This makes them well suited for an empirical test of theoretical isochrones like in the study that White et al. (1999) have done for the young quadruple system GG Tau.

The semimajor axes of $\mathrm{V} 773$ Tau $\mathrm{AB}$ and $\mathrm{AC}$ have probably the same order of magnitude, which means that V 773 Tau ABC is not a hierarchical triple system. This poses the question if the whole system is dynamically stable. This is 


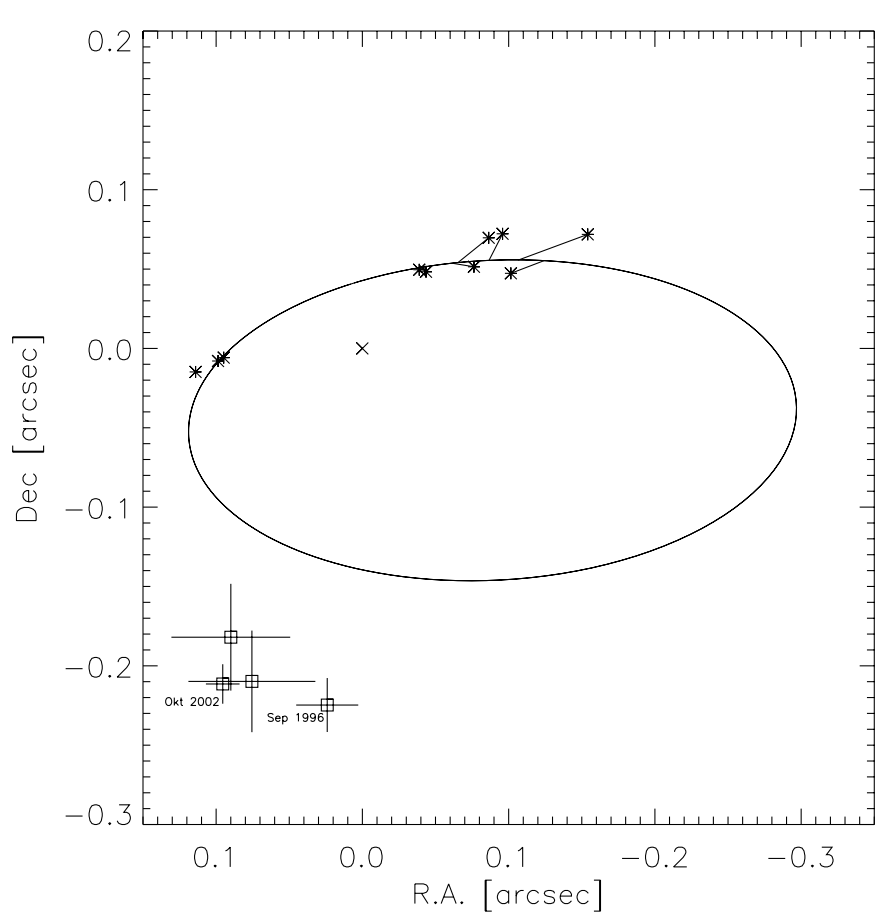

Fig. 2. Orbital motion in the V 773 Tau system. The cross at $(0.0$, 0.0 ) marks the primary V 773 Tau A. The asterisks denote the relative positions of V 773 Tau B together with the orbital fit by Tamazian et al. (2002). The squares represent relative position measurements of the new companion V 773 Tau C from September 1996 (most right) to October 2002 (most left).

probably the case. Although age determinations from pre-main sequence evolutionary models are highly uncertain, one can fairly assume that V 773 Tau is at least some $10^{5} \mathrm{yr}$ old since it is a Class III source with no signs of infalling envelopes or strong circumstellar accretion. This age is much larger than the orbital periods of the companions (125 yr for V 773 Tau AB, Tamazian et al. 2002), so if there were dynamical instabilities they would have already destroyed the system. It remains however unclear why this is not the case. Another striking property of the V 773 Tau system seen in Fig. 2 is that both visual companions apparently move in different directions on the sky. This means that they either do not orbit in the same direction or have quite different orbital inclinations. For a better understanding of this situation additional observations will be needed to include V 773 Tau C into the orbit calculation for V 773 Tau AB by Tamazian et al. (2002) and then derive the orbital parameters of the whole triple system.

The detection of only one additional companion has of course no significant impact on binary statistics. The finding of an additional component in a very well studied young multiple system like V 773 Tau however indicates that there may be even more companion stars in Taurus-Auriga and that higher order multiple systems may be more common among pre-main sequence stars than was previously thought. The known binary stars in Taurus-Auriga and other SFRs are for this reason interesting targets for next-generation high angular resolution instruments that will be able to close the detection gap between spectroscopic and visual companions.

Acknowledgements. The author acknowledges a thoughtful and constructive report from the anonymous referee that led to a significant improvement of this paper. J.W. is supported from the Deutsches Zentrum für Luft- und Raumfahrt under grant number 50 OR 0009. The observations on Calar Alto were supported by travel money from the Deutsche Forschungsgemeinschaft (grant numbers Wo 834 / 11, Wo 834 / 2-1 and Wo 834 / 4-1). The data reduction has been performed using the "Binary/Speckle" package developed by Rainer Köhler. I am also grateful to the night assistants and the technical staff on Calar Alto for their support during my observing runs.

\section{References}

Duchêne, G. 1999, A\&A, 341, 547

Duchêne, G., Ghez, A. M., \& McCabe, C. 2001, BAAS, 199, 8906

Duquennoy, A., \& Mayor, M. 1991, A\&A, 248, 455

Ghez, A. M., Neugebauer, G., \& Matthews, K. 1993, AJ, 106, 2005

Herbig, G. H., \& Bell, K. R. 1988, Lick Observatory Bulletin, 1111

Kenyon, S. J., \& Hartmann, L. W. 1995, ApJS, 101, 117

Knox, K. T., \& Thompson, B. J. 1974, ApJ, 193, L45

Köhler, R., \& Leinert, Ch. 1998, A\&A, 331, 977

Köhler, R., Kunkel, M., Leinert, Ch., \& Zinnecker, H. 2000, A\&A, 356,541

Leinert, Ch., Zinnecker, H., Weitzel, N., et al. 1993, A\&A, 278, 129

Lestrade, J. F., Preston, R. A., Jones, D. L., et al. 1999, A\&A, 344, 1014

Lohmann, A. W., Weigelt, G., \& Wirnitzer, B. 1983, Appl. Opt., 22, 4028

Mathieu, R. D., Ghez, A. M., Jensen, E., \& Simon, M. 2000, in Protostars and Planets IV, ed. V. Mannings, A. P. Boss, \& S. S. Russell (Tucson, Arizona: The University of Arizona Press)

McCaughrean, M. J., \& Stauffer, J. R. 1994, AJ, 108, 1382

Richichi, A., Köhler, R., Woitas, J., \& Leinert, Ch. 1999, A\&A, 364, 501

Tamazian, V. S., Docobo, J. A., White, R. J., \& Woitas, J. 2002, ApJ, 578, 925

Welty, A. D. 1995, AJ 110, 776

White, R. J., Ghez, A. M., Reid, I. N., \& Schultz, G. 1999, ApJ, 520, 811

Woitas, J., Köhler, R., \& Leinert, Ch. 2001, A\&A, 369, 249 